\title{
A universal fixture design of box-type equipments on warship board DONG Zhen-jie,ZHENG Chen-yao
}

\author{
Army of 91388, Zhanjiang, Guangdong, CHINA \\ 1137004735@qq.com
}

Keywords: screw kit; box-type equipment; fixture; angle iron; anti-corrosion; work platform

\begin{abstract}
In this paper we provide a universal fixture design procedure of shipboard box-type equipments, the basic principle is to manufacture two sets of screw kits with angle iron devices, install L-type hook structures (or F-type structure) at both ends of the kits, then fix on the work platform according to the width of the box-type equipment. We rotate screws to realize adjusting measurement to adapt to length of the device. The angle iron devices achieve the function of fixing the entire box-type equipment. The fixture measurement can be adjusted which ensure the safety and normal use of the box-type equipment, and guarantee the successful development of the test.
\end{abstract}

\section{Introduction}

During the seaborne test processes of warships, the unfavorable objective conditions, such as changeable climate, poor environment and small space, may lead to complicated test work and affect the advance of tasks. Because of the hull's unavoidable swing and bump during warships navigation, especially when there is a large storm and the sea conditions is poor, the shipboard box-type equipments often will slide, displace or even overturn which will bring about the equipments' abnormal operations even damages, it will immediately affect tests' smooth development. Most of the warship holds are relatively narrow in consideration of the design, while this is exactly the place where most of operations of the test equipments are carried out. Safety and security are hard to be realized by manpower. Therefore it is urgent to design fixtures of warships box-type equipments on board $^{[1]}$.

Most of warships don't specially design box-type equipment fixtures at the participation in seaborne tests, all equipments are temporarily transported to the shipboard without dedicated fixtures to match them. Therefore it is important to design universal fixtures which can meet the seaborne tests' safety and security of various kinds of equipments, the operation is simple and convenient as well.

\section{Key points of design}

According to the problems that may occur during use of the ship borne box-type equipment, we summarize several requirements that should be paid attention to when designing the fixture as follows:

Fixtures must be carried easily and move conveniently with small volume. The fixture is too large that will affect use of the box-type equipment and bring inconvenience to the operation because of narrow space on the shipboard. Meanwhile the fixture changes positions along with the box-type equipment which requiring easily to be carried; The versatility of fixtures must be stronger which can adjust measurement to meet fixed requirement of box-type equipments with different size or thickness. We ensure the thickness depend on the height of both screw kits and angle iron; Fixtures should easily operate. Because time is life in the course of tests, the fixture isn't easy to be handled that will delay time and bring unnecessary losses about tests; Fixtures should have anti-corrosion features. In order to adapt to the harsh environment of high temperature, high humidity and high salt on sea, the simply constructed fixtures must have certain anti-corrosion features to ensure long-term and repeated use without damages; Fixtures should have certain strength. We can ensue the effective fixing of box-type equipments only the strength is high enough, otherwise the fixtures are easily bended or even damaged, and safety of the box-type equipments can't be guaranteed ${ }^{[2]}$. 


\section{Recommendations of selecting material}

We offer proposals of selecting material when designing fixtures: Choose metal material to design fixtures which has a larger strength, lower cost. The metal material is easy to process, modify and rework, but the quality is too large, inconvenient to carry and not resistant to corrosion. It can meet the anti-corrosion requirement to a certain degree through a special treatment; Choose nonmetal material to design fixtures which has a high corrosion property, light weight, and easy to carry. We consider making mould in the step of design which has higher cost, and not easy to carry, but can be used for forming mass production ${ }^{[3]}$.

According to the characteristics of each material above the fixture selects the metal material in the design stage, that is, a steel fixture. After the fixture effect is verified, then we choose nonmetal material to make mould and mass production, provide to shipboard which ensure the safety use of test equipments.

\section{Production process of fixtures}

In the warship's cabin, since the box-type equipment generally places on the plane work platform, then the fixture must first fixes on the work platform that can fix the box-type equipment. In consideration of different warships' cabin equip with different sizes of the work platform, the fixtures require adjustable size of length and width.

The universal fixture's design and production process of the box-type equipment is as follows:

The first step: Design and manufacture the screw kits. We choose making screw form because of the fixture requires an adjustable size, prepare two cylindrical steel rods and two long screws, tap in the middle of each steel rod, make an internal thread to match with the external thread. The screws are inserted into the center of the steel rods to form two screw kits. Each outer is a sliding sleeve, the internal is a long screw. We install L-type hook structures at both ends of the kits to fix the screws onto the plane work platform, and realize adjustable length through rotating the thread. The adjust range can custom design according to specific requirements.

The second step: Weld sliding angle iron devices. Since most box-type equipments are similar in shape to cuboids, we consider fix the two device diagonals to realize fixing the whole box-type equipment. The two angle irons are welded to the two sliding sleeve of screw kits which can move following sliding sleeves' motion, and realize the manual and adjustable length. One screw kit with angle iron device is shown in Figure 1 through drawing with $3 \mathrm{Dmax}^{[4]}$.

As shown in the figure 1, the two ends of the screw kit are fixed with an L-type hook structure or an inverted F-type structure. The latter has better stability than the former, so the actual production chooses the inverted F-type structure.

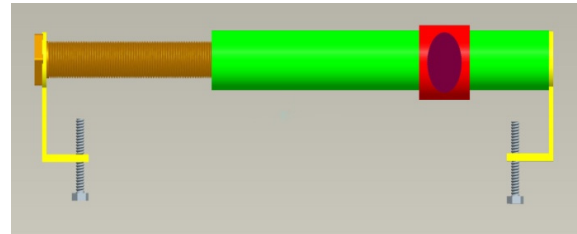

Figure 1 the screw kit with angle iron device

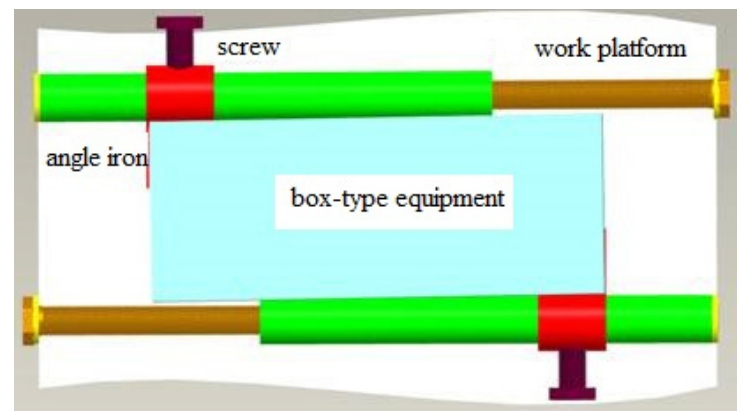

Figure 2 installation of universal fixtures of the box-type equipments

The third step: Set positions of the two screw kits. We fix the two screw kits onto the plane work platform, adjust the L-type structures in accordance with the width of the devices, control screw kits to adapt to the width of the box-type equipment's size, and slide angle irons to adjust the length, then fix the two diagonal corners of equipment, so that the equipment can be firmly fixed on the plane work platform, won't slide or shift in the swinging and bumpy cabin. In order to increase the friction, the inner side of the angle irons are pasted anti-skidding rubber mat which will buffer and protect the box-type equipment. Since the angle iron has a certain height, then the fixed box-type equipment 
won't overturn or damage due to tilt of the hull. The schematic diagram of installation of the fixture is shown in Figure 2.

\section{Fixture simulation}

In order to further verify the feasibility, effectiveness, universality and adaptability of the fixture we must verify the actual case. Because the laboratory's operating platform resembles a plane work platform inside the warship's cabin where it is tested by fixing the box-type equipment. The box-type equipment places on the simulation platform, adjusts the distance between two screw kits according to the width of device, fixes the two ends of screw kits with the inverted F-type structure, rotate the screw kits to achieve the length adjustment to adapt the equipment's length. The angle irons firmly lock the two diagonal corners. We paste a rubber pad inside the angle iron which ensures that the contact equipment area don't abrade and the friction will increase at the same time to make the equipment more firm.

First we make two kinds of structures using in the fixture:

The two screw kits with angle irons. The screw kits with angle irons are shown in Figure 3. Wherein the length of cylinder steel rod is $L_{1}=550 \mathrm{~mm}$, the diameter is $R_{1}=26 \mathrm{~mm}$, the left end is the screw kit, the length is $L_{2}=100 \mathrm{~mm}$, the diameter is $R_{2}=18 \mathrm{~mm}$. the angle iron device is shown in Figure 4 . The angle iron is welded to the cylinder sleeve which slides on the steel rod, the size of the angle iron is $50 \mathrm{~mm} * 50 \mathrm{~mm}$. The sleeve has a small screw for fixing the angle iron device and the screw kit. Since the screw can rotate and adjust the length of the kit, then the theoretical length of the screw kit is $0 \sim 650 \mathrm{~mm}$. Due to the structural design of the angle iron device, the maximum sliding can not reach the edge of the screw kit, the actual range is $0 \sim 630 \mathrm{~mm}$.

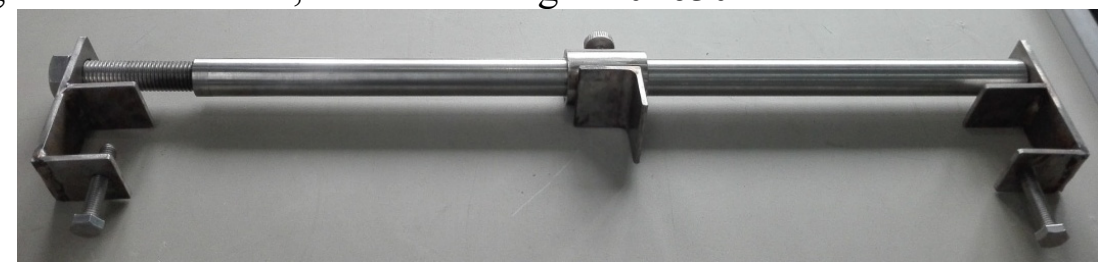

Figure 3 the physical map of the screw kit with angle irons

The inverted F-type structures. The inverted F-type structures are installed on both ends of the screw kits. the real object is shown in Figure 5. Wherein the height of C-type bayonet is $50 \mathrm{~mm}$, the small screw below can adjust the bayonet height though rotating to achieve fixing the work platform of different thickness.
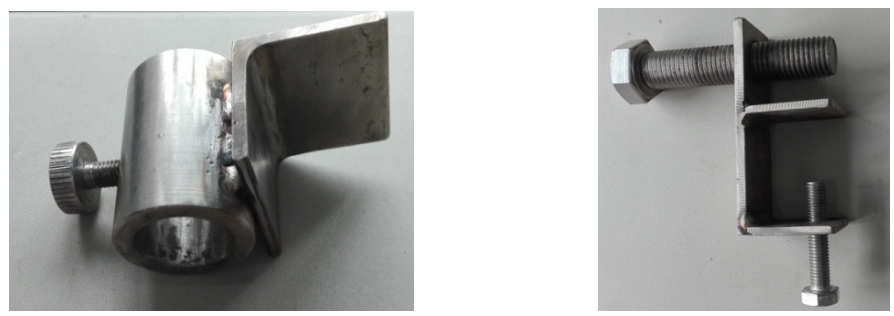

Figure 4 the physical map of the angle iron device Figure 5 the physical map of the inverted F-type structure

Then test and verify by utility model:

The two different sizes of box-type equipments are placed on the work platform, adjust the distance between the two screw kits, fix the inverted F-type structures on the work platform, and fix the equipment from the width direction, and then slide the angle iron devices, so that the inside of the two angle irons is close to the two diagonal corners of the box-type equipment, as shown respectively in Figure 6(a,b). The dimensions of the two box-type equipments in the picture are respectively $300 \mathrm{~mm} * 200 \mathrm{~mm} * 120 \mathrm{~mm}$ and $450 \mathrm{~mm} * 300 \mathrm{~mm} * 250 \mathrm{~mm}$. 

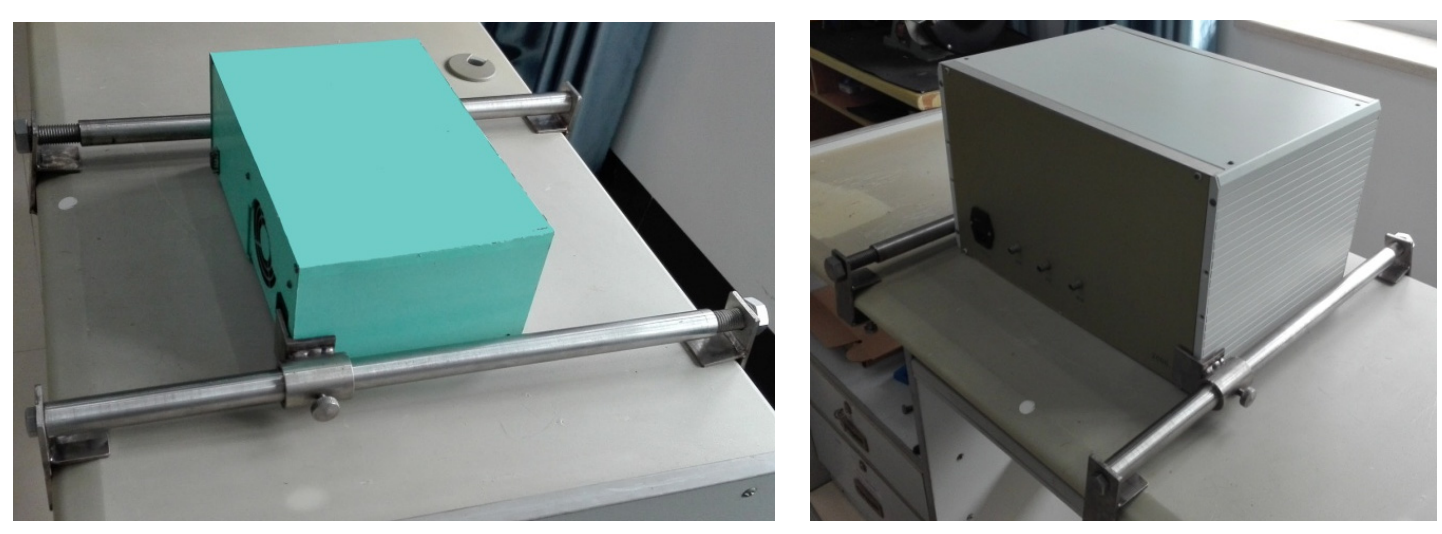

Figure $6(\mathrm{a}, \mathrm{b})$ the physical map of fixtures applying to two box-type equipments with different sizes

It is seen from Figure 6 that the fixture can fix the box-type equipments with different sizes, the range of width is not limited, and the range of length is $0 \sim 630 \mathrm{~mm}$ which can fix the common box-type equipments. It verifies the generality, reliability and effectiveness, relieves security risks of the box-type equipments' displacement aboard on warships. The location of the angle iron which fixes the box-type equipment is at the bottom. The angle iron is high enough to save steels, the fixing effect is also good. But for the higher height of box-type equipments, we consider adjusting the contact position between angle irons and equipments, such as the middle position, increase the height of the inverted F-type structure to lift the location of the angle irons, and achieve fixing higher equipments.

\section{Summary}

The whole fixture is made of steels which fully meet the requirements of the hardness of fixing box-type equipments. The steels surface has the anti-corrosion measures to ensure a long-term reuse of the fixture. As generally many box-type equipments are on shipboard at the same time, then each equipment requires equipping with a fixture. In consideration of the portability and generality of the fixture we design the fixture model by non-metallic materials in mass production. The fixtures that are produced in this way meets the requirement of hardness, corrosion-resistant and portable. We need that the maximum adjustment size of the screw kit is larger for fixing a small amount of large-scale box-type equipments. The diameter of cylinder steel rods and screws which we choose in the design must be increased to meet the fixing requirement. It verifies the reform ability of the fixture.In view of the fixing of box-type equipments are from the pre-test preparation to the end of the test, and then enchase the equipments, a long time metal contact and extrusion may make the equipments' protective surface wear or off, stick a rubber pad inside the angle iron which increases friction in the buffer extrusion and enhances the fixing effect.

\section{References}

[1] Chen zhan, Wang chunfei, Ying keman. Study on simple medical treatment fixture on warship board, J. Clinical medicine, vol. 5, pp. 633-634, 2007.

[2] Lu fengyi, Zhong shouyan. M, Machine Design. Beijing: Machinery industry press, 2011.

[3] Lu yaozu, Zheng huiqiang. M, Machine structure Design. Shanghai: Tong ji university press, 2009.

[4] Ye yuju, Jiao yonghe, Zhang tong. M, Machine drawing manual. Beijing:, Machinery industry press, 2012. 